\title{
Reconstruction of Liouvillian Superoperators
}

\author{
Vladimír Bužek \\ Institute of Physics, Slovak Academy of Sciences, Dúbravská cesta 9, 84228 Bratislava, Slovakia
}

(February 16, 1998)

\begin{abstract}
We show how to determine (reconstruct) a master equation governing the time evolution of an open quantum system. We present a general algorithm for the reconstruction of the corresponding Liouvillian superoperators. Dynamics of a two-level atom in various environments is discussed in detail.
\end{abstract}

Proper description of quantum dynamics of open systems is essential for our understanding of physical processes in many areas of physics, starting from quantum optics to quantum cosmology. In general an open system can be represented as a system $S$ interacting with an environment $E$ [1]. In this paper we consider the archetypal system + environment model which is specified as follows: Let $\mathcal{H}_{S}$ denotes a Hilbert space of the system $S$, and $\mathcal{H}_{E}$ is the Hilbert space associated with the environment $E$. The Hamiltonian $\hat{H}_{S E}=$ $\hat{H}_{S} \otimes \hat{1}_{E}+\hat{H}_{i n t}+\hat{1}_{S} \otimes \hat{H}_{E}$ of the composite system $S \oplus E$ acts on $\mathcal{H}_{S} \otimes \mathcal{H}_{E}$. It is assumed that $S \oplus E$ is a closed finite-dimensional system which evolves unitarily. The density operator $\hat{\rho}_{S E}(t)$ of this composite system is governed by the von Neumann equation with the formal solution $\hat{\rho}_{S E}(t)=\exp \left[-i\left(t-t_{0}\right) \hat{H}_{S E}\right] \hat{\rho}_{S E}\left(t_{0}\right) \exp \left[i\left(t-t_{0}\right) \hat{H}_{S E}\right]$, where the initial state is $\hat{\rho}_{S E}\left(t_{0}\right)=\hat{\rho}_{S}\left(t_{0}\right) \otimes \hat{\rho}_{E}\left(t_{0}\right)$ and $\hbar=1$. The reduced dynamics of the system $S$ is then defined as

$$
\hat{\rho}_{S}(t):=\hat{\mathcal{T}}\left(t, t_{0}\right) \hat{\rho}_{S}\left(t_{0}\right)=\operatorname{Tr}_{E}\left[\hat{\rho}_{S E}(t)\right] .
$$

By definition, $\hat{\mathcal{T}}\left(t, t_{0}\right)$ is a linear map which transforms the input state $\hat{\rho}_{S}\left(t_{0}\right)$ onto the output state $\hat{\rho}_{S}(t)$. In this paper we address the question how to determine (reconstruct) the master equation which governs the time evolution of the reduced density operator $\hat{\rho}_{S}(t)$. This master equation can be written in the convolutionless form [2] (we omit the subscript $S$ )

$$
\frac{d}{d t} \hat{\rho}(t)=\hat{\mathcal{L}}\left(t, t_{0}\right) \hat{\rho}(t)
$$

which is possible due to the fact that in the finitedimensional Hilbert spaces matrix elements of density operators are analytic functions. Consequently, $\hat{\mathcal{T}}\left(t, t_{0}\right)$ are non-singular operators (except may be for a set of isolated values of $t$ ) in which case the inverse operators $\hat{\mathcal{T}}\left(t, t_{0}\right)^{-1}$ exist and the Liouvillian superoperator can be expressed as

$$
\hat{\mathcal{L}}\left(t, t_{0}\right):=\left[\frac{d}{d t} \hat{\mathcal{T}}\left(t, t_{0}\right)\right] \hat{\mathcal{T}}^{-1}\left(t, t_{0}\right) .
$$

We note that $\hat{\mathcal{T}}\left(t, t_{0}\right)$ is uniquelly specified by $\hat{H}_{S E}$ and by the initial state $\hat{\rho}_{E}\left(t_{0}\right)$ of the environment.

In this paper we propose a general algorithm how to reconstruct the Liouvillian superoperator $\hat{\mathcal{L}}\left(t, t_{0}\right)$ from the the knowledge of the time evolution of the system density operator $\hat{\rho}(t)$. In fact there are two aspects of this problem. Firstly, $\hat{\rho}(t)$ can be given as a result of a sequence of quantum-tomography measurements [3], such that at each time $t$ the system density operator is reconstructed from the measured tomographic data. From these experimental data then the Liouvillian which governs the open system can be reconstructed (see Example $A$ ). Secondly, the density operator $\hat{\rho}(t)$ is determined from the knowledge of the unitary evolution of the composite $S \oplus E$ system [see Eq.(11)]. From this knowledge the master equation (2) is determined. In both cases the dynamics of the open system is given exclusively in terms of the system operators. Environmental degrees of freedom are completely eliminated from the reduced dynamics. Nevertheless, the state of the environment may change during the time evolution due to the interaction with the system. That is, we do not employ the assumption that the environment is a "big" reservoir which does not change under the action of the system (see Examples $B$ and $C$ ).

In order to reconstruct the Liouvillian superoperator $\hat{\mathcal{L}}\left(t, t_{0}\right)$ we have to determine firstly the linear map $\hat{\mathcal{T}}\left(t, t_{0}\right)$ given by Eq.(11). This part of the reconstruction can be performed with the help of the algorithm recently proposed by Poyatos, Cirac and Zoller [⿶. This algorithm works as follows: Let us assume that the system $S$ has been initially prepared in a pure state $\left|\Psi\left(t_{0}\right)\right\rangle=\sum_{i_{1}=0}^{N} c_{i_{1}}\left|i_{1}\right\rangle$ where $\left|i_{1}\right\rangle$ are basis vectors in the $(N+1)$-dimensional Hilbert space $\mathcal{H}_{S}$ of the system under consideration. It is further assumed that the environment is initially prepared in a state $\hat{\rho}_{E}\left(t_{0}\right)=$ $\sum_{\alpha_{1} \alpha_{2}} d_{\alpha_{1} \alpha_{2}}\left|\alpha_{1}\right\rangle_{E}\left\langle\alpha_{2}\right|$, where $\left|\alpha_{i}\right\rangle_{E}$ are basis vectors in the Hilbert space $\mathcal{H}_{E}$ of the environment.

In general, the physical process $\hat{\mathcal{T}}\left(t_{k}, t_{0}\right)$ is determined by a transformation acting on basis vectors of the system and the environment (in what follows we omit in all expressions the explicit reference to the initial time $t_{0}$ )

$$
\left|i_{1}\right\rangle_{S}\left|\alpha_{1}\right\rangle_{E} \stackrel{\hat{\mathcal{T}}\left(t_{k}\right)}{\longrightarrow} \sum_{j_{1}=0}^{N} \sum_{\beta_{1}} E_{\left(i_{1} j_{1}\right)\left(\alpha_{1} \beta_{1}\right)}\left(t_{k}\right)\left|j_{1}\right\rangle_{S}\left|\beta_{1}\right\rangle_{E} .
$$

The output density operator $\hat{\rho}\left(t_{k}\right)$ of the system at time $t_{k}$ is obtained when the transformation (14) is applied to the initial state of the system-environment $\hat{\rho}\left(t_{0}\right) \otimes \hat{\rho}_{E}\left(t_{0}\right)$ and then the tracing over the environment is performed, so that $\hat{\rho}\left(t_{k}\right)$ can be written as 


$$
\hat{\rho}\left(t_{k}\right)=\sum_{i_{1}, i_{2}=0}^{N} c_{i_{1}}\left(c_{i_{2}}\right)^{*} \hat{R}_{\left(i_{1}, i_{2}\right)}\left(t_{k}\right),
$$

where $(N+1)^{2}$ operators $\hat{R}_{\left(i_{1}, i_{2}\right)}\left(t_{k}\right)$ are defined as

$$
\hat{R}_{\left(i_{1}, i_{2}\right)}\left(t_{k}\right)=\sum_{j_{1}, j_{2}=0}^{N} D_{\left(i_{1}, i_{2}\right)\left(j_{1}, j_{2}\right)}\left(t_{k}\right)\left|j_{1}\right\rangle\left\langle j_{2}\right|,
$$

with

$$
\begin{aligned}
D_{\left(i_{1}, i_{2}\right)\left(j_{1}, j_{2}\right)}\left(t_{k}\right) & =\sum_{\alpha_{1}, \alpha_{2}, \gamma} d_{\alpha_{1} \alpha_{2}} \\
& \times E_{\left(i_{1} j_{1}\right)\left(\alpha_{1} \gamma\right)}\left(t_{k}\right) E_{\left(i_{2} j_{2}\right)\left(\alpha_{2} \gamma\right)}^{*}\left(t_{k}\right) .
\end{aligned}
$$

From Eq.(5) it follows that the process $\hat{\mathcal{T}}\left(t_{k}\right)$ for a given time $t_{k}$ is completely determined by $(N+1)^{2}$ operators $\hat{R}_{\left(i_{1}, i_{2}\right)}\left(t_{k}\right)$, which in turn are specified by the $(N+1)^{2} \times(N+1)^{2}$ matrix elements $D_{\left(i_{1}, i_{2}\right)\left(j_{1}, j_{2}\right)}\left(t_{k}\right)$. We note that $\hat{R}_{\left(i_{1}, i_{2}\right)}\left(t_{k}\right)$ have the properties

$$
\begin{aligned}
& \operatorname{Tr} \hat{R}_{\left(i_{1}, i_{2}\right)}\left(t_{k}\right)=\delta_{i_{1}, i_{2}} ; \\
& \left(\hat{R}_{\left(i_{1}, i_{2}\right)}\left(t_{k}\right)\right)^{\dagger}=\hat{R}_{\left(i_{2}, i_{1}\right)}\left(t_{k}\right),
\end{aligned}
$$

or, equivalently,

$$
\begin{aligned}
\sum_{j=0}^{N} D_{\left(i_{1}, i_{2}\right)(j, j)}\left(t_{k}\right) & =\delta_{i_{1}, i_{2}} ; \\
D_{\left(i_{1}, i_{2}\right)\left(j_{1}, j_{2}\right)}^{*}\left(t_{k}\right) & =D_{\left(i_{2}, i_{1}\right)\left(j_{2}, j_{1}\right)}\left(t_{k}\right) .
\end{aligned}
$$

We also note that neither $\hat{R}_{\left(i_{1}, i_{2}\right)}\left(t_{k}\right)$ nor $D_{\left(i_{1}, i_{2}\right)\left(j_{1}, j_{2}\right)}\left(t_{k}\right)$ depend on the initial state $\hat{\rho}\left(t_{0}\right)$ of the system and formally they fulfill the conditions

$$
\begin{aligned}
\lim _{t_{k} \rightarrow t_{0}} \hat{R}_{\left(i_{1}, i_{2}\right)}\left(t_{k}\right) & =\left|i_{1}\right\rangle\left\langle i_{2}\right| ; \\
\lim _{t_{k} \rightarrow t_{0}} D_{\left(i_{1}, i_{2}\right)\left(j_{1}, j_{2}\right)}\left(t_{k}\right) & =\delta_{i_{1}, j_{1}} \delta_{i_{2}, j_{2}} .
\end{aligned}
$$

Poyatos et al. [1] have shown that in order to specify the $(N+1)^{2}$ operators $\hat{R}_{\left(i_{1}, i_{2}\right)}\left(t_{k}\right)$ one has to consider $(N+1)^{2}$ specific (see below) initial conditions $\left|\Psi^{\left(k_{1}, k_{2}\right)}\right\rangle_{\text {in }}=\sum_{i_{1}=0}^{N} c_{i_{1}}^{\left(k_{1}, k_{2}\right)}\left|i_{1}\right\rangle$ where $k_{1}, k_{2}=0,1, \ldots, N$ and to measure the corresponding $(N+1)^{2}$ output density operators $\hat{\rho}^{\left(k_{1}, k_{2}\right)}\left(t_{k}\right)$ which can be expressed as

$$
\hat{\rho}^{\left(k_{1}, k_{2}\right)}\left(t_{k}\right)=\sum_{i_{1}, i_{2}=0}^{N} M_{\left(k_{1}, k_{2}\right)\left(i_{1}, i_{2}\right)} \hat{R}_{\left(i_{1}, i_{2}\right)}\left(t_{k}\right),
$$

where

$$
M_{\left(k_{1}, k_{2}\right)\left(i_{1}, i_{2}\right)}=c_{i_{1}}^{\left(k_{1}, k_{2}\right)}\left(c_{i_{2}}^{\left(k_{1}, k_{2}\right)}\right)^{*} .
$$

If the $(N+1)^{2}$ initial conditions $\left|\Psi^{\left(k_{1}, k_{2}\right)}\right\rangle_{\text {in }}$ are chosen so, that the matrix $M_{\left(k_{1}, k_{2}\right)\left(i_{1}, i_{2}\right)}$ given by Eq. (12) is invertible, then the set of Eqs.(11) can be solved with respect of the operators $\hat{R}_{\left(i_{1}, i_{2}\right)}\left(t_{k}\right)$. Alternatively, one can express the matrix elements $D_{\left(i_{1}, i_{2}\right)\left(j_{1}, j_{2}\right)}\left(t_{k}\right)$ as functions of the in and out states of the measured system, i.e.

$$
\begin{array}{r}
D_{\left(i_{1}, i_{2}\right)\left(j_{1}, j_{2}\right)}\left(t_{k}\right)= \\
\sum_{k_{1}, k_{2}=0}^{N} \tilde{M}_{\left(i_{1}, i_{2}\right)\left(k_{1}, k_{2}\right)} S_{\left(k_{1}, k_{2}\right)\left(j_{1}, j_{2}\right)}\left(t_{k}\right),
\end{array}
$$

where the $(N+1)^{2} \times(N+1)^{2}$ matrix $S$ is defined as

$$
S_{\left(k_{1}, k_{2}\right)\left(j_{1}, j_{2}\right)}\left(t_{k}\right)=\left\langle j_{1}\left|\hat{\rho}^{\left(k_{1}, k_{2}\right)}\left(t_{k}\right)\right| j_{2}\right\rangle .
$$

The matrix $\tilde{M}$ is the inverse of $M$ and has the property $\sum_{k_{1}, k_{2}=0} \tilde{M}_{\left(j_{1}, j_{2}\right)\left(k_{1}, k_{2}\right)} M_{\left(k_{1}, k_{2}\right)\left(i_{1}, i_{2}\right)}=\delta_{i_{1}, j_{1}} \delta_{i_{2}, j_{2}}$. So this is how the process $\hat{\mathcal{T}}\left(t_{k}\right)$ can be reconstructed from the measured in and out states. To make the reconstruction possible the matrix $M$ has to be invertible. Obviously, there are many choices of such matrix. In particular, Poyatos et al. [4] have proposed $M$ given by Eq.(12) with complex amplitudes $c_{i}^{\left(k_{1}, k_{2}\right)}$ specified as

$$
c_{i}^{\left(k_{1}, k_{2}\right)}=\left\{\begin{array}{ll}
\left(\delta_{i, k_{1}}+\delta_{i, k_{2}}\right) / \sqrt{2} & \text { if } k_{1}>k_{2} \\
\delta_{i, k_{1}} & \text { if } k_{1}=k_{2} \\
\left(\delta_{i, k_{1}}+i \delta_{i, k_{2}}\right) / \sqrt{2} & \text { if } k_{1}<k_{2}
\end{array} .\right.
$$

The reconstruction process described above gives us a set of operators $\hat{R}_{\left(i_{1}, i_{2}\right)}\left(t_{k}\right)$ which describe the transition of the system from the state $\hat{\rho}\left(t_{0}\right)$ to the state $\hat{\rho}\left(t_{k}\right)$ at a given time $t_{k}$. In principle, one can perform a whole sequence of such reconstructions at different times $t_{1}, t_{2}, \ldots . t_{K}$ so that the reduced dynamics of the studied system can be reconstructed from the measured data.

Now our task is to determine (reconstruct) from a set of measurements of the output states $\hat{\rho}^{\left(k_{1}, k_{2}\right)}(t)$ for given input states $\hat{\rho}^{\left(k_{1}, k_{2}\right)}\left(t_{0}\right)$, the form of the Liouvillian superoperator $\hat{\mathcal{L}}(t)$ in Eq.(2). To do so, we firstly note, that when the time evolution of the operators $\hat{\rho}^{\left(k_{1}, k_{2}\right)}(t)$ is governed by Eq.(2), then taking into account the expression (11) and the assumption that the matrix $M$ is invertible, we find that the operators $\hat{R}_{\left(i_{1}, i_{2}\right)}(t)$ are also governed by the same master equation, i.e.

$$
\frac{d}{d t} \hat{R}_{\left(i_{1}, i_{2}\right)}(t)=\hat{\mathcal{L}}(t) \hat{R}_{\left(i_{1}, i_{2}\right)}(t),
$$

with the initial conditions given by Eq.(10). Alternatively, taking into account the expression (6) we obtain from Eq.(16) a set of linear differential equations for matrix elements $D_{\left(i_{1}, i_{2}\right)\left(k_{1}, k_{2}\right)}(t)$

$$
\frac{d}{d t} D_{\left(i_{1}, i_{2}\right)\left(k_{1}, k_{2}\right)}(t)=
$$

$$
\sum_{j_{1}, j_{2}=0}^{N} D_{\left(i_{1}, i_{2}\right)\left(j_{1}, j_{2}\right)}(t) G_{\left(j_{1}, j_{2}\right)\left(k_{1}, k_{2}\right)}(t),
$$

with the initial conditions (10). Here the matrix $G_{\left(j_{1}, j_{2}\right)\left(k_{1}, k_{2}\right)}(t)$ is defined as 


$$
G_{\left(j_{1}, j_{2}\right)\left(k_{1}, k_{2}\right)}(t)=\left\langle k_{1}\left|\left(\hat{\mathcal{L}}(t)\left|j_{1}\right\rangle\left\langle j_{2}\right|\right)\right| k_{2}\right\rangle,
$$

and it uniquely determines the Liouvillian superoperator $\hat{\mathcal{L}}(t)$.

We already know how to reconstruct matrices $D$ from the measured data for arbitrary time $t$ (from these data we can also evaluate the corresponding time derivatives). Providing the matrix $D_{\left(i_{1}, i_{2}\right)\left(j_{1}, j_{2}\right)}(t)$ is not singular its inverse $\tilde{D}_{\left(j_{1}, j_{2}\right)\left(i_{1}, i_{2}\right)}(t)$ can be found and then the reconstructed matrix $G_{\left(j_{1}, j_{2}\right)\left(k_{1}, k_{2}\right)}(t)$ is given by a simple expression

$$
\begin{array}{r}
G_{\left(j_{1}, j_{2}\right)\left(k_{1}, k_{2}\right)}(t)= \\
\sum_{i_{1}, i_{2}=0}^{N} \tilde{D}_{\left(j_{1}, j_{2}\right)\left(i_{1}, i_{2}\right)}(t) \frac{d}{d t} D_{\left(i_{1}, i_{2}\right)\left(k_{1}, k_{2}\right)}(t)
\end{array}
$$

from which the superoperator $\hat{\mathcal{L}}(t)$ at time $t$ can be determined. This is the main result of the paper.

In the following we will apply this general algorithm into three physically interesting examples.

Example A. - Let us consider a two-level system (a twolevel atom, a spin-1/2, or a qubit) with a two-dimensional Hilbert space $\mathcal{H}_{S}$ spanned by two vectors $|1\rangle$ and $|0\rangle$. In order to specify the Liouvillian superoperator $\hat{\mathcal{L}}(t)$ for the two-level atom we have to know the time evolution of four initial states specified by Eq.(15). Let us assume that from the measured data it is found that these states evolve as

$$
\begin{aligned}
\hat{\rho}^{(0,0)}(t) & =\left(\begin{array}{ll}
0 & 0 \\
0 & 1
\end{array}\right) ; \quad \hat{\rho}^{(1,1)}(t)=\left(\begin{array}{cc}
e^{-\Gamma t} & 0 \\
0 & 1-e^{-\Gamma t}
\end{array}\right) . \\
\hat{\rho}^{(0,1)}(t) & =\frac{1}{2}\left(\begin{array}{cc}
e^{-\Gamma t} & i e^{-\Gamma t / 2} \\
-i e^{-\Gamma t / 2} & 2-e^{-\Gamma t}
\end{array}\right) ; \\
\hat{\rho}^{(1,0)}(t) & =\frac{1}{2}\left(\begin{array}{cc}
e^{-\Gamma t} & e^{-\Gamma t / 2} \\
e^{-\Gamma t / 2} & 2-e^{-\Gamma t}
\end{array}\right) .
\end{aligned}
$$

Now we can apply our reconstruction scheme and we find for the matrix $G_{\left(j_{1}, j_{2}\right)\left(k_{1}, k_{2}\right)}(t)$ the expression [5]

$$
G_{\left(j_{1}, j_{2}\right)\left(k_{1}, k_{2}\right)}(t)=\left(\begin{array}{cccc}
-\Gamma & 0 & 0 & \Gamma \\
0 & -\Gamma / 2 & 0 & 0 \\
0 & 0 & -\Gamma / 2 & 0 \\
0 & 0 & 0 & 0
\end{array}\right)
$$

This matrix corresponds to the Liouvillian which defines the master equation

$$
\frac{d}{d t} \hat{\rho}=\hat{\mathcal{L}} \hat{\rho}=\frac{\Gamma}{2}\left[2 \hat{\sigma}_{-} \hat{\rho} \hat{\sigma}_{+}-\hat{\sigma}_{+} \hat{\sigma}_{-} \hat{\rho}-\hat{\rho} \hat{\sigma}_{+} \hat{\sigma}_{-}\right],
$$

describing the decay of a two-level atom into a zerotemperature reservoir [6]. The Liouvillian in Eq.(22) is time independent which reflects the fact that the state of the reservoir does not change in time under the influence of the system.

Example B. - Here we will reconstruct the Liouvillian superoperator for the master equation describing the time evolution of a single two-level atom interacting with a single-mode electro-magnetic field in an ideal cavity. The corresponding Hamiltonian in the dipole and the rotating-wave approximations reads [6]

$$
\hat{H}=\omega_{A} \hat{\sigma}_{z}+\omega \hat{a}^{\dagger} \hat{a}+\lambda\left(\hat{\sigma}_{+} \hat{a}+\hat{\sigma}_{-} \hat{a}^{\dagger}\right)
$$

where $\lambda$ is the atom-field coupling constant. We assume that the atomic transition frequency $\left(\omega_{A}\right)$ is on the resonance with the filed frequency $(\omega)$. The operators $\hat{a}^{\dagger}$ and $\hat{a}$ are the usual photon creation and annihilation operators, respectively, with $\left[\hat{a}, \hat{a}^{\dagger}\right]=1$. If the atom and the field are initially prepared in states $\left|\Psi\left(t_{0}\right)\right\rangle_{A}=$ $c_{0}|0\rangle+c_{1}|1\rangle$, and $\left|\Psi\left(t_{0}\right)\right\rangle_{F}=\sum_{k=0} e_{k}|k\rangle \equiv|\alpha\rangle$, respectively, then at time $t$ the atom-field state vector $|\Psi(t)\rangle_{A-F}$ reads

$$
\begin{array}{r}
|\Psi(t)\rangle_{A-F}=c_{0} \sum_{k}\left(\cos \tau_{k}|k\rangle|0\rangle-i \sin \tau_{k}|k-1\rangle|1\rangle\right) \\
\quad+c_{1} \sum_{k}\left(\cos \tau_{k+1}|k\rangle|1\rangle-i \sin \tau_{k+1}|k+1\rangle|0\rangle\right),
\end{array}
$$

where $\tau_{k}=\lambda \sqrt{k} t$. Utilizing Eq.(19) we can determine the Liouvillian superoperator which governs the dynamics of the atom. Here $\hat{\mathcal{L}}(t)$ explicitly depends on the initial state of the cavity field. Let us assume a particular case when the field has been prepared in the Fock state $|M\rangle$. With this initial state the matrix (13) takes the form

$$
D_{\left(i_{1}, i_{2}\right)\left(j_{1}, j_{2}\right)}(t)=\left(\begin{array}{cccc}
\xi_{1} & 0 & 0 & 1-\xi_{1} \\
0 & \sqrt{\xi_{0} \xi_{1}} & 0 & 0 \\
0 & 0 & \sqrt{\xi_{0} \xi_{1}} & 0 \\
1-\xi_{0} & 0 & 0 & \xi_{0}
\end{array}\right)
$$

where $\xi_{0}=\cos ^{2}(\lambda t \sqrt{M})$ and $\xi_{1}=\cos ^{2}(\lambda t \sqrt{M+1})$. The determinant of this matrix $\operatorname{det}[D]=\xi_{0} \xi_{1}\left(\xi_{0}+\xi_{1}-1\right)$ is equal to zero only at discrete moments so $D$ is invertible and we can utilize Eq.(19) from which we find

$$
G_{\left(j_{1}, j_{2}\right)\left(k_{1}, k_{2}\right)}(t)=\left(\begin{array}{cccc}
-\gamma_{1} & 0 & 0 & \gamma_{1} \\
0 & -\gamma_{2} / 2 & 0 & 0 \\
0 & 0 & -\gamma_{2} / 2 & 0 \\
\gamma_{3} & 0 & 0 & -\gamma_{3}
\end{array}\right)
$$

with the time-dependent parameters $\gamma_{i}(t)$ given as

$$
\gamma_{1}(t)=\frac{2 \lambda\left(\sqrt{M} \sin (2 \lambda t \sqrt{M}) \sin ^{2}(\lambda t \sqrt{M+1})+\sqrt{M+1} \sin (2 \lambda t \sqrt{M+1}) \cos ^{2}(\lambda t \sqrt{M})\right)}{[\cos (2 \lambda t \sqrt{M})+\cos (2 \lambda t \sqrt{M+1})]}
$$




$$
\begin{aligned}
& \gamma_{2}(t)=\frac{\lambda\left(\sqrt{M} \sin (2 \lambda t \sqrt{M}) \cos ^{2}(\lambda t \sqrt{M+1})+\sqrt{M+1} \sin (2 \lambda t \sqrt{M+1}) \cos ^{2}(\lambda t \sqrt{M})\right)}{\cos ^{2}(\lambda t \sqrt{M}) \cos ^{2}(\lambda t \sqrt{M+1})} \\
& \gamma_{3}(t)=\frac{2 \lambda\left(\sqrt{M} \sin (2 \lambda t \sqrt{M}) \cos ^{2}(\lambda t \sqrt{M+1})+\sqrt{M+1} \sin (2 \lambda t \sqrt{M+1}) \sin ^{2}(\lambda t \sqrt{M})\right)}{[\cos (2 \lambda t \sqrt{M})+\cos (2 \lambda t \sqrt{M+1})]} .
\end{aligned}
$$

From the solution (26) it follows that the Liouvillian superoperator is explicitly time-dependent which reflects dynamical response of the environment (i.e. the cavity field). The master equation (2) with $\hat{\mathcal{L}}(t)$ specified by Eq.(26) can be written as

$$
\begin{aligned}
\frac{d}{d t} \hat{\rho} & =\frac{\gamma_{1}(t)}{2}\left[2 \hat{\sigma}_{-} \hat{\rho} \hat{\sigma}_{+}-\hat{\sigma}_{+} \hat{\sigma}_{-} \hat{\rho}-\hat{\rho} \hat{\sigma}_{+} \hat{\sigma}_{-}\right] \\
& -\frac{\eta(t)}{2}\left[\hat{\sigma}_{+} \hat{\sigma}_{-} \hat{\rho} \hat{\sigma}_{-} \hat{\sigma}_{+}+\hat{\sigma}_{-} \hat{\sigma}_{+} \hat{\rho} \hat{\sigma}_{+} \hat{\sigma}_{-}\right] \\
& +\frac{\gamma_{3}(t)}{2}\left[2 \hat{\sigma}_{+} \hat{\rho} \hat{\sigma}_{-}-\hat{\sigma}_{-} \hat{\sigma}_{+} \hat{\rho}-\hat{\rho} \hat{\sigma}_{-} \hat{\sigma}_{+}\right],
\end{aligned}
$$

with the coefficients $\gamma_{i}(t)$ Eq.(27) and $\eta(t)=\gamma_{2}(t)-$ $\gamma_{1}(t)-\gamma_{3}(t)$. One can check that $\hat{\rho}_{A}(t)$ obtained from Eq.(24) is the solution of the master equation (28). We note that if the cavity field is initially in the vacuum state $(M=0)$ then the master equation (28) takes the form (22) but with the time-dependent "decay" rate $\Gamma \rightarrow \gamma_{1}(t)=2 \lambda \tan \lambda t$.

Example C. - Finally, we consider a single two-level atom coupled to $K$ modes of the electro-magnetic field in a one-dimensional cavity of the length $L$. The spectrum of modes is discrete with frequencies $\omega_{k}=k \pi c / L$. The corresponding total Hamiltonian in the dipole and rotating-wave approximations reads [7]

$$
\hat{H}=\omega_{A} \hat{\sigma}_{z}+\sum_{k=1}^{K} \omega_{k} \hat{a}_{k}^{\dagger} \hat{a}_{k}+\sum_{k=1}^{K} \lambda_{k}\left(\hat{\sigma}_{+} \hat{a}_{k}+\hat{\sigma}_{-} \hat{a}_{k}^{\dagger}\right) .
$$

The field is assumed to be initially in the vacuum state. By applying our algorithm we find the master equation for the atom to be of the form (22) except the "decay" rate $\Gamma \rightarrow \gamma(t)$ is now explicitly time dependent. It can be expressed in terms of the "measured" probability $P(t)=\left\langle 1\left|\hat{\rho}_{A}(t)\right| 1\right\rangle$ that the upper atomic level is excited:

$$
\gamma(t)=-\left(\frac{d P(t)}{d t}\right) P(t)^{-1}
$$

In Fig.11 we present the time evolution of $P(t)$ and $\gamma(t)$ obtained with the help of numerical diagonalization of the Hamiltonian (29). From our results it follows that $\gamma(t=0)=0$ but as soon as the atom starts to radiate the function $\gamma(t)$ starts to grow and after a short time it takes the constant value $\Gamma=2 \pi \lambda^{2} d_{\text {eff }}(\omega)$ given by the Golden Fermi rule [7]. At this stage the atom radiates exponentially and two wave packets propagating to the left and the right cavity mirrors are irradiated. These packets are reflected by mirrors at $t=L / 2 c$ and they "kick" back the atom at $t=L / c$. At this point the atom is essentially in its ground state and the reflected waves packets (environment) force it to absorb energy, i.e. the atom does not decay exponentially anymore. This is the reason why during the recurrence of the atomic inversion $\gamma(t)$ rapidly changes and takes negative values.

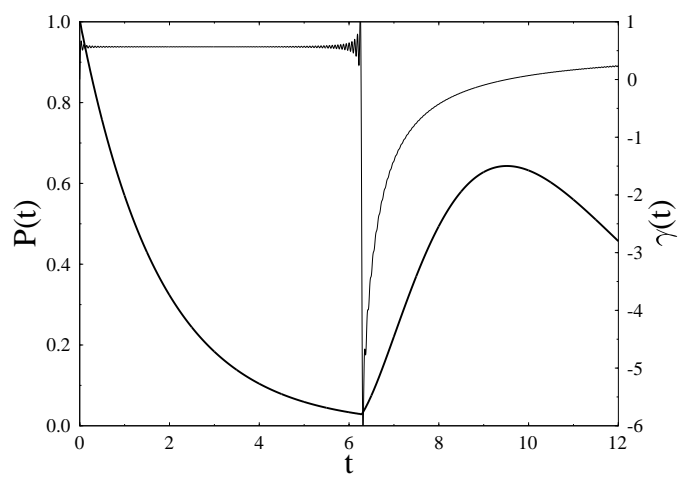

FIG. 1. The time evolution of the decay rate $\gamma(t)$ (thin line) and the population of the excited atomic level $P(t)$ (thick line). We assume the atom to be in the center of the 1-D cavity, so it is coupled only to the odd modes (i.e. $\lambda_{2 k}=0$ ). We assume $L=2 \pi$ and $c=1$ so that $\omega_{2 k+1}=k+1 / 2$, and $\lambda_{2 k+1}=\lambda=0.3$. The effective density of modes which interact with the atom is $d_{e f f}(\omega)=L / 2 c \pi=1$. Therefore the decay rate $\Gamma=2 \pi \lambda^{2} d_{\text {eff }}(\omega) \simeq 0.564$. We consider $K=400$ modes of the field initially in the vacuum state and the atom (with $\omega_{A}=101$ ) in its upper state $|1\rangle$.

I thank Pavel Bóna, Ignacio Cirac, Gabriel Drobný, Jason Twamley, and Peter Zoller for helpful discussions and comments. This work was supported by the $A k$ tion Österreich - Slowakische Republik under the project $18 \mathrm{~s} 42$, and by the Royal Society.

[1] E.B. Davies, Quantum Theory of Open Systems (Academic Press, London, 1976); for futher recent references see, for instance, A. Royer, Phys. Rev. Lett. 77, 3272 (1996).

[2] P. Bóna, private communication

[3] U. Leonhardt, Phys. Rev. Lett. 74, 4101 (1995).

[4] J.F. Poyatos, J.I. Cirac, and P. Zoller, Phys. Rev. Lett. 78, 390 (1997).

[5] Matrix elements $G_{\left(j_{1}, j_{2}\right)\left(k_{1}, k_{2}\right)}$ are label so, that the pair of $\left(j_{1}, j_{2}\right)$ indicate lines [ the $j$-th line is numbered as $\left.j=(N+1)^{2}-(N+1) j_{1}-j_{2}\right]$ while $\left(k_{1}, k_{2}\right)$ indicate rows [with $k$-th row numbered as $k=(N+1)^{2}-(N+1) k_{1}-k_{2}$ ].

[6] W.H. Louisell, Quantum Statistical Properties of Radiation (Wiley, New York, 1973).

[7] C. Cohen-Tanoudji, et al., Atom-Photon Interactions: Basic Processes and Applications (Wiley, New York, 1992). 